\title{
Two Mutually Loss-coupled Lasers Featuring Astable Multivibrator
}

\author{
A. Mustafin \\ Department of Physics, University of Maryland, College Park, MD 20742, USA
}

\begin{abstract}
Self-sustained antiphase relaxation oscillations of high amplitude are shown to be possible in a system of two single-mode semiconductor lasers strongly coupled through their cavities.
\end{abstract}

Key words: coupled lasers, second-order loss, antiphase state

PACS: 05.45.Xt, 42.65.Sf, 87.23.Cc

\section{Introduction}

It has been known that individual single mode laser does not exhibit selfsustained oscillations. However self-pulsing regimes of different complexity are well possible in coupled lasers. Recently synchronization in coupled semiconductor lasers has been a subject of extensive study not only because laser is one of the important nonlinear dynamical systems, but also owing to potential applications of synchronization phenomena to communication, electronic circuits and even biological systems.

The overwhelming majority of synchronization schemes employ either various modifications of optoelectronic cross-coupling [1a-c], or face-to-face mutual coupling $[2 \mathrm{a}-\mathrm{c}]$. In the former scheme, the output of each laser is detected and converted into an electronic signal by a photodetector, and after amplification the signal is fed back to modulate the pump current of the other laser. In the latter scheme, the output of each laser is injected, after a suitable attenuation, in the other laser. While handling the above two types of coupling both theoretically and experimentally in numerous papers, a whole series of interesting effects has been discovered. Under certain conditions, a time delay introduced by the mutual feedback being paramount, coupling can drive semiconductor lasers into nonlinear oscillations, such as regular pulsing, quasi-periodic pulsing, or chaotic pulsing. 
At the same time, there is perhaps the only work dealing with an alternative mechanism of intensity coupling based on cross-loss [3]. In their version of such a coupling scheme, an additional loss in either laser is induced by macroscale mechanical deformations of the crystal structure in a common support due to strong local heating. Consideration based on the equations proposed by [4] with added intensity-dependent losses has shown a self-pulsing instability of the steady state solutions.

There is good reason to believe that cross-loss coupling harbors a great deal of interesting synchronization effects highly competitive in diversity with those of the known coupling schemes. However the thermomechanical embodiment of this principle suffers from the slowness of heating in relation to the dynamics of photons and carriers. Signal exchange via thermomechanical modulation of the crystal structure cannot be sped up by placing the beams closer to each other because experimentalist has to avoid field-field interaction. More promising way is to use one or other type of intracavity q-modulator controlled by electric, magnetic, or acoustic pulses.

In the present paper a system of two semiconductor lasers coupled in such a manner that cavity loss rate of each one is proportional to the output of its counterpart, is studied in terms of the rate equations for photon and carrier densities. Another key assumption of the model is existence of a nonzero second-order cavity loss associated with two-photon absorption at high powers. The plausible range for that second-order loss rate is estimated. The existence of a self-pulsing regime featured by spiky antiphase relaxation oscillations is inferred from the system of coupled rate equations by applying multiple-scale approximation techniques. Any time delay in coupling is not a prerequisite for the emergence of synchronous oscillations. The frequency of the obtained antiphase-locked pulsing is shown to be considerably lower than that of intrinsic underdamped quasi-harmonic oscillations of a standalone laser and completely independent of the concrete value of the second-order loss rate.

\section{Rate equations of a single-mode laser}

We take, as the starting point, the following rate equations for a single-mode semiconductor laser:

$$
\begin{aligned}
& \dot{P}=\left(G(N)-\gamma_{p}-D P\right) P, \\
& \dot{N}=\frac{J}{e d}-G(N) P-\gamma_{n} N .
\end{aligned}
$$

(The dot denotes $d / d t$.) Here $P$ and $N$ are the respective densities of photon and carrier population inversion inside the laser cavity. The cavity is assumed 
to have thickness $d$. Linear function of carriers, $G(N)=\Gamma v_{\mathrm{g}} a\left(N-N_{0}\right)$, is the net rate of stimulated emission, where $\Gamma$ is the confinement factor (the ratio of the volume of the cavity to the volume occupied by photons in the cavity), $v_{\mathrm{g}}$ is the light group velocity, $a$ is the gain constant, and $N_{0}$ is the carrier density at transparency. $\gamma_{p}=v_{\mathrm{g}}\left(\alpha_{\mathrm{f}}+\alpha_{\text {int }}\right)$ is the photon loss rate due to both facet $\left(\alpha_{\mathrm{f}}\right)$ and internal $\left(\alpha_{\text {int }}\right)$ losses. $J$ is the density of a pump current flowing through the active region and $e$ is the elementary charge. $\gamma_{n}$ is the carrier loss rate solely due to nonradiative effects. Somewhat simplifying the picture, we assume the contribution of both radiative and Auger recombination loss mechanisms negligible. Taking those into account does not qualitatively affect our ensuing results, however makes consideration more involved.

An additional (small) quadratic loss term $D P^{2}$ is introduced in the first equation of (1) to allow for nonlinear mechanism of photon fluctuations damping, such as two-photon absorption, anticipated at high powers.

The meaning and typical values of the different parameters (mostly borrowed from [5]) in the model (1) are given in Table 1. The same numerical values of the parameters are used in the calculations. Equations (1) can be converted into dimensionless form by performing the linear scaling

$$
\begin{array}{ll}
p=P \Gamma v_{g} a \gamma_{n}^{-1}, & n=\left(N-N_{0}\right) \Gamma v_{g} a \gamma_{p}^{-1}-1, \\
\delta=D \gamma_{n}\left(\Gamma v_{g} a \gamma_{p}\right)^{-1}, & j=\left(J\left(e d \gamma_{n}\right)^{-1}-N_{0}\right) \Gamma v_{g} a \gamma_{p}^{-1}-1, \\
\varepsilon=\gamma_{n} / \gamma_{p}, &
\end{array}
$$

and they become

$$
\begin{aligned}
& \dot{p}=\varepsilon^{-1} \gamma_{n}(n-\delta p) p, \\
& \dot{n}=\gamma_{n}(j-(n+1) p-n) .
\end{aligned}
$$

Note that now the dimensionless $n$ and $j$ are no longer proportional to their dimensional prototypes, but rather are deviations of the corresponding absolute quantities from the threshold of generation. Value of $\varepsilon$ representing the ratio of the time constants of the two equations is of order $O\left(10^{-4}\right)$ because the rate constants $\gamma_{p}$ and $\gamma_{n}$ differ almost ten thousand times.

Seemingly transparent, the dynamics of system (3) is worth touching briefly on to estimate how small the hypothetical second-order cavity loss might be and to provide more seamless passage to the coupled dynamics to be treated in the next section. 
Table 1

Typical parameter values for a single-mode uncoupled laser

\begin{tabular}{|c|c|c|c|}
\hline Parameter & Meaning & Value & Units \\
\hline$c$ & speed of light in vacuum & $3 \cdot 10^{10}$ & $\mathrm{~cm} / \mathrm{s}$ \\
\hline$e$ & elementary charge & $1.6 \cdot 10^{-19}$ & $\mathrm{C}$ \\
\hline$\mu_{\mathrm{g}}$ & group refractive index & 4 & - \\
\hline$v_{\mathrm{g}}=c / \mu_{\mathrm{g}}$ & light group velocity & $0.75 \cdot 10^{10}$ & $\mathrm{~cm} / \mathrm{s}$ \\
\hline$\Gamma$ & confinement factor & 0.3 & - \\
\hline$a$ & gain constant & $2.5 \cdot 10^{-16}$ & $\mathrm{~cm}^{2}$ \\
\hline$d$ & cavity thickness & $2 \cdot 10^{-5}$ & $\mathrm{~cm}$ \\
\hline$N_{0}$ & carrier density at transparency & $10^{18}$ & $\mathrm{~cm}^{-3}$ \\
\hline$\gamma_{n}$ & nonradiative carrier loss rate & $10^{8}$ & $\mathrm{~s}^{-1}$ \\
\hline$\alpha_{\mathrm{f}}$ & facet cavity loss & 45 & $\mathrm{~cm}^{-1}$ \\
\hline$\alpha_{\text {int }}$ & internal cavity loss & 40 & $\mathrm{~cm}^{-1}$ \\
\hline$\gamma_{p}$ & cavity loss rate & $0.6375 \cdot 10^{12}$ & $\mathrm{~s}^{-1}$ \\
\hline$J$ & pump current density & $5 \cdot 10^{3}$ & $\mathrm{~A} / \mathrm{cm}^{2}$ \\
\hline$j$ (eq. (2)) & dimensionless pump & 11.90 & - \\
\hline$D$ & second-order cavity loss & $\begin{array}{l}2.954 \cdot 10^{-6} \\
\text { (conditional) }\end{array}$ & $\mathrm{cm}^{3} / \mathrm{s}$ \\
\hline$\delta($ eq. $(2))$ & dimensionless second-order cavity loss & $0.8239 \cdot 10^{-3}$ & - \\
\hline$\varepsilon($ eq. $(2))$ & ratio of the time constants & $1.569 \cdot 10^{-4}$ & - \\
\hline$T_{\text {intr }}($ eq. $(6))$ & period of intrinsic oscillations & $2.281 \cdot 10^{-10}$ & $\mathrm{~s}$ \\
\hline
\end{tabular}

Two physically meaningful steady states of system (3) are possible in the $(p, n)$ phase plane (Fig. 1):

$$
\begin{aligned}
& p^{(1)}=0, \quad n^{(1)}=j ; \\
& p^{(2)}=\left(\sqrt{(1+\delta)^{2}+4 \delta j}-1-\delta\right) /(2 \delta)=j-\delta j(j+1)+O\left(\delta^{2}\right), \\
& n^{(2)}=\left(\sqrt{(1+\delta)^{2}+4 \delta j}-1-\delta\right) / 2=\delta j+O\left(\delta^{2}\right) .
\end{aligned}
$$

Steady state 1 is always a saddle since eigenvalues $\lambda$ of the Jacobian matrix $\mathbf{J}$ of $(3)$, being the roots of the characteristic polynomial $\operatorname{det}(\mathbf{J}-\lambda \mathbf{I})=\lambda^{2}-$ $(\operatorname{tr} \mathbf{J}) \lambda+\operatorname{det} \mathbf{J}$, have opposite signs: $\lambda_{1}=-\gamma_{n}, \lambda_{2}=j \gamma_{n} / \varepsilon$. 


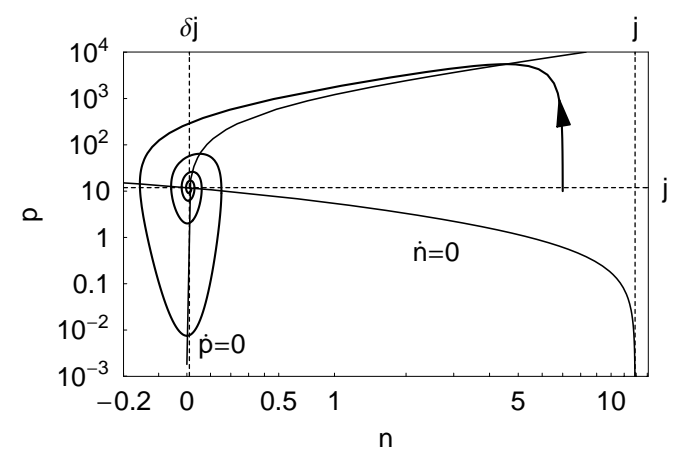

(a)

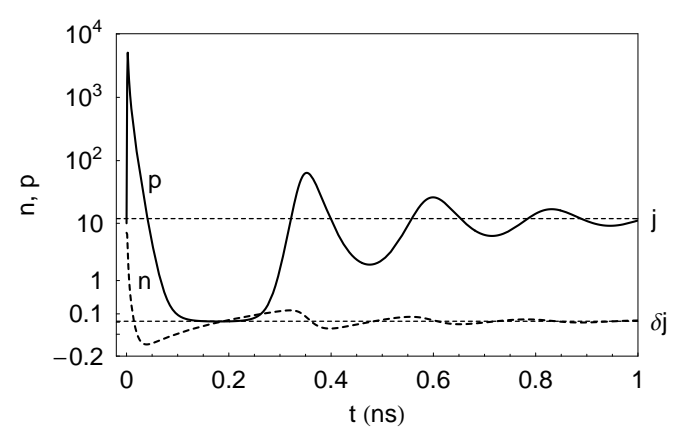

(b)

Fig. 1. (a) Phase portrait of a single laser corresponding to system (3). Steady states lie at the intersections of nullclines $\dot{p}=0$ and $\dot{n}=0$. Steady state with nonzero population inversion is a stable focus, while the steady state on the $n$-axis is a saddle. (b) Time profiles of underdamped intrinsic oscillations (solid: photons, dashed: carriers).

Steady state 2 is always a stable focus/node, because $\operatorname{tr} \mathbf{J}=-1-p^{(2)}-$ $\varepsilon^{-1} \delta p^{(2)}<0$ and $\operatorname{det} \mathbf{J}=\varepsilon^{-1} \gamma_{n}^{2} p^{(2)}\left(1+\delta\left(1+2 p^{(2)}\right)\right)>0$. Normally, it must be a focal point, because it is just transient intrinsic underdamped oscillations that could be experimentally observed in a semiconductor laser briefly after turning it on [6]. For steady state 2 to be a focus, the discriminant of the quadratic characteristic equation has to be negative: $\Delta=(\operatorname{tr} \mathbf{J})^{2}-4 \operatorname{det} \mathbf{J}<0$. Correct to the second order in $\delta$, this condition can be reduced to

$$
\Delta=\varepsilon^{-2} \gamma_{n}^{2} j\left(\delta^{2} j-4 \varepsilon\right)<0
$$

Solving the above inequality yields an upper bound for $\delta$ :

$$
\delta<\delta_{\max }=2 \sqrt{\varepsilon / j} .
$$

For a laser with typical $j$ and $\varepsilon$ given in Table 1 , the magnitude of $\delta_{\max }$ is $0.726 \cdot 10^{-2}$.

While $\delta$ remains such that $\delta=o\left(\varepsilon^{1 / 2}\right)$, the second-order loss has a negligible effect on the period of the intrinsic oscillations, for which we get

$$
T_{\mathrm{intr}}=4 \pi|\Delta|^{-1 / 2}=2 \pi \gamma_{n}^{-1} \sqrt{\varepsilon / j}
$$

Numerically, this is 228 ps.

The lower-bound estimate for the second-order loss can be performed by studying the decay time of the intrinsic oscillations. The decay time is the inverse of the damping factor, which in turn is a half of the trace of the Jacobian 
matrix: $\tau_{\text {dec }}=2|\operatorname{tr} \mathbf{J}|^{-1}$. Keeping only the first terms in the expansion of $\operatorname{tr} \mathbf{J}$ in powers of $\delta$ yields $\tau_{\text {dec }}=2 \varepsilon \gamma_{n}^{-1}(\delta j+\varepsilon(j+1))^{-1}$.

Two different asymptotic cases are possible. In the first case $\delta=o(\varepsilon)$. This condition is stronger than (5). We then obtain $\tau_{\text {dec }}=2 \gamma_{n}^{-1}(j+1)^{-1}$. The result means that in the absence of the second-order cavity loss the decay time is totally determined by the carriers' recombination rate and must be of the order of several nanoseconds.

In the alternative case $\varepsilon=o(\delta)$. Now $\tau_{\text {dec }}=2 \varepsilon\left(\gamma_{n} \delta j\right)^{-1}$, so the intrinsic oscillations may decay $\delta / \varepsilon$ times faster. This is a noticeable difference contributed by the second-order damping. It should be mentioned, that C.J. Kennedy and J.D. Barry [7] were first to indicate the importance of the second-order damping although the damping in their laser system had a different physical nature, resulting from the intracavity frequency doubling. It is precisely this interplay between the two small parameters in the system, $\varepsilon$ and $\delta$, that is of crucial importance in determining the temporal hierarchy of a laser. If we are given some experimentally observed maximum characteristic time of decay, $\tau_{\text {decmax }}$, then the lower bound for $\delta$ can be found from the condition $\tau_{\text {dec }}<\tau_{\text {decmax }}$ resulting in

$$
\delta>\delta_{\min }=\varepsilon\left(2 /\left(\gamma_{n} \tau_{\text {decmax }}\right)-j-1\right) / j .
$$

It has been known from the experiments [5] that intrinsic oscillations in semiconductor lasers do decay much faster than the standard rate equations involving only first-order cavity loss predict. We can ascribe this extra damping to the second-order cavity loss. The observed $\tau_{\text {decmax }}$ is usually shorter than a nanosecond. Hence for the typical values of $j$ and $\varepsilon, \delta_{\min }$ would be $0.935 \cdot 10^{-4}$. For the present we cannot judge with any confidence about the value of $\delta$, except of it must be related to $\varepsilon$ via conditions (5) and (7). Nevertheless, as we will demonstrate further, the exact value of $\delta$ is not all that critical, and all the results would remain valid providing this parameter is within the wide range from (7) through (5). For the purposes of model calculations, we adopted $\delta$ to be a geometric mean of $\delta_{\min }$ and $\delta_{\max }$, i.e. $0.8239 \cdot 10^{-3}$.

An alternative way to introduce the effective second-order cavity loss in a model is to assume that the material gain has a linear dependence not only on the carrier but also on the photon density, as is done in the work of R. Vicente et al. [8].

Since the small parameter multiplies the derivative $\dot{p}$, system (3) is singularly perturbed (e.g. [9]). The significant difference between carrier and photon lifetimes brings multiscale properties into the model. It contains relatively fast variable, $p$, and slow variable, $n$. However, the hasty conclusion that $\gamma_{n}^{-1}$ and $\gamma_{p}^{-1}$ are some unique characteristic times of fluctuations of the corresponding 
variables would be an oversimplification. Indeed, at any current value of $n$ the fluctuations of $p$ have a characteristic time of order $\varepsilon / n$. Thus, in the vicinity of the focal point the difference in rates of change of the two variables is not as great as when far from the steady state.

\section{Two loss-coupled lasers: statics}

Consider a pair of not necessarily identical, but having comparable parameters, lasers of type (1) cross-coupled through their resonators, so that each of them can modulate cavity loss of the other:

$$
\begin{aligned}
& \dot{P}_{1}=\left(G_{1}\left(N_{1}\right)-\gamma_{p_{1}}-D_{1} P_{1}-K_{2} P_{2}\right) P_{1}, \\
& \dot{P}_{2}=\left(G_{2}\left(N_{2}\right)-\gamma_{p_{2}}-D_{2} P_{2}-K_{1} P_{1}\right) P_{2}, \\
& \dot{N}_{1}=J_{1} /\left(e d_{1}\right)-G_{1}\left(N_{1}\right) P_{1}-\gamma_{n_{1}} N_{1}, \\
& \dot{N}_{2}=J_{2} /\left(e d_{2}\right)-G_{2}\left(N_{2}\right) P_{2}-\gamma_{n_{2}} N_{2} .
\end{aligned}
$$

Here $K_{1}$ and $K_{2}$ are positive coupling strengths. On the proper rescaling, this system takes the following form:

$$
\begin{aligned}
& \dot{p}_{1}=\varepsilon_{1}^{-1} \gamma_{n_{1}}\left(n_{1}-\delta_{1} p_{1}-\varkappa_{2} p_{2}\right) p_{1} \\
& \dot{p}_{2}=\varepsilon_{2}^{-1} \gamma_{n_{2}}\left(n_{2}-\delta_{2} p_{2}-\varkappa_{1} p_{1}\right) p_{2} \\
& \dot{n}_{1}=\gamma_{n_{1}}\left(j_{1}-\left(n_{1}+1\right) p_{1}-n_{1}\right) \\
& \dot{n}_{2}=\gamma_{n_{2}}\left(j_{2}-\left(n_{2}+1\right) p_{2}-n_{2}\right) .
\end{aligned}
$$

The dimensionless coupling strengths $\varkappa_{1,2}=K_{1,2} \gamma_{n_{1,2}}\left(\gamma_{p_{2,1}} \Gamma_{1,2} v_{\mathrm{g} 1,2} a_{1,2}\right)^{-1}$ do not have to be weak; we assume that at least,

$$
\varkappa_{1,2} \gg \delta_{1,2} \text {. }
$$


Model (8) has four steady states. To $O(1)$ for small $\delta_{1,2}$,

$$
\begin{aligned}
p_{1}^{(1)} & =j_{1}, \quad p_{2}^{(1)}=0, \quad n_{1}^{(1)}=0, \quad n_{2}^{(1)}=j_{2} ; \\
p_{1}^{(2)} & =0, \quad p_{2}^{(2)}=j_{2}, \quad n_{1}^{(2)}=j_{1}, \quad n_{2}^{(2)}=0 ; \\
p_{1}^{(3)} & =0, \quad p_{2}^{(3)}=0, \quad n_{1}^{(3)}=j_{1}, \quad n_{2}^{(3)}=j_{2} ; \\
p_{1}^{(4)} & =\left(1-\varkappa_{1}\left(j_{1}+\varkappa_{2}\right)+\varkappa_{2} j_{2}-Q\right) /\left(2 \varkappa_{1}\left(\varkappa_{2}-1\right)\right) \\
& =j_{1}-\varkappa_{2} j_{2}\left(j_{1}+1\right)+O\left(\varkappa_{1}^{2}+\varkappa_{2}^{2}\right), \\
p_{2}^{(4)} & =\left(1-\varkappa_{2}\left(j_{2}+\varkappa_{1}\right)+\varkappa_{1} j_{1}-Q\right) /\left(2 \varkappa_{2}\left(\varkappa_{1}-1\right)\right) \\
& =j_{2}-\varkappa_{1} j_{1}\left(j_{2}+1\right)+O\left(\varkappa_{1}^{2}+\varkappa_{2}^{2}\right), \\
n_{1}^{(4)} & =\varkappa_{2} p_{2}^{(4)}=\varkappa_{2} j_{2}+O\left(\varkappa_{1}^{2}+\varkappa_{2}^{2}\right), \\
n_{2}^{(4)} & =\varkappa_{1} p_{1}^{(4)}=\varkappa_{1} j_{1}+O\left(\varkappa_{1}^{2}+\varkappa_{2}^{2}\right), \\
Q & =\left(\left(1+\varkappa_{2} j_{2}-\varkappa_{1}\left(j_{1}+\varkappa_{2}\right)\right)^{2}+4 \varkappa_{1}\left(\varkappa_{2}-1\right)\left(\varkappa_{2} j_{2}-j_{1}\right)\right)^{1 / 2} .
\end{aligned}
$$

Steady states 1 and 2 are "pure" in the sense that either of them corresponds to one device lasing while the other being inactive. Correct to $O(1)$ in $\varepsilon_{1,2}$, the eigenvalues for steady state 1 are $\lambda_{1}=-\gamma_{n_{2}}, \lambda_{2}=-\varepsilon_{2}^{-1} \gamma_{n_{2}}\left(\varkappa_{1} j_{1}-j_{2}\right)$, $\lambda_{3,4}=-\gamma_{n_{1}}\left(\left(j_{1}+1\right) / 2 \pm \mathrm{i} \sqrt{j_{1} / \varepsilon_{1}}\right)$; whence it follows that this equilibrium is stable when

$$
\varkappa_{1}>j_{2} / j_{1}
$$

Similar reasoning shows that steady state 2 is stable when

$$
\varkappa_{2}>j_{1} / j_{2}
$$

Steady states 3 and 4 are "mixed" in the sense that either of them corresponds to both lasers being in the same mode of operation. In steady state 3 both lasers are inactive (do not emit any light). This steady state is always unstable, because two of the four associated eigenvalues are positive: $\lambda_{1}=-\gamma_{n_{1}}, \lambda_{2}=$ $-\gamma_{n_{2}}, \lambda_{3}=\varepsilon_{1}^{-1} \gamma_{n_{1}} j_{1}, \lambda_{4}=\varepsilon_{2}^{-1} \gamma_{n_{2}} j_{2}$.

"Mixed" steady state 4, wherein both lasers are active, exists in the positive quadrant only if $\varkappa_{1}<j_{2} / j_{1}$ and $\varkappa_{2}<j_{1} / j_{2}$. Note, that if this steady state is physically feasible, then both "pure" steady states, 1 and 2 , are unstable. The necessary and sufficient conditions for all the eigenvalues of the Jacobian matrix, evaluated at steady state 4 , to have negative real parts, are, from the Routh-Hurwitz criterion, 


$$
\begin{aligned}
c_{1} & >0 \\
c_{1} c_{2}-c_{3} & >0 \\
\left(c_{1} c_{2}-c_{3}\right) c_{3}-c_{1}^{2} c_{4} & >0 \\
c_{4} & >0
\end{aligned}
$$

where $c_{1}, c_{2}, c_{3}$ and $c_{4}$ are the coefficients of the characteristic polynomial $\lambda^{4}+c_{1} \lambda^{3}+c_{2} \lambda^{2}+c_{3} \lambda+c_{4}$ :

$$
\begin{aligned}
c_{1}= & \gamma_{n_{1}}\left(p_{1}^{(4)}+1\right)+\gamma_{n_{2}}\left(p_{2}^{(4)}+1\right), \\
c_{2}= & \varepsilon_{1}^{-1} \varepsilon_{2}^{-1}\left(\varepsilon_{2} \gamma_{n_{1}}^{2} p_{1}^{(4)}+\varepsilon_{1} \gamma_{n_{2}}^{2} p_{2}^{(4)}+\left(\varepsilon_{2} \varkappa_{2} \gamma_{n_{1}}^{2}+\varepsilon_{1} \varkappa_{1} \gamma_{n_{2}}^{2}\right) p_{1}^{(4)} p_{2}^{(4)}\right. \\
& \left.+\varepsilon_{1} \varepsilon_{2} \gamma_{n_{1}} \gamma_{n_{2}}\left(p_{1}^{(4)}+p_{2}^{(4)}+1\right)+\gamma_{n_{1}} \gamma_{n_{2}}\left(\varepsilon_{1} \varepsilon_{2}-\varkappa_{1} \varkappa_{2}\right) p_{1}^{(4)} p_{2}^{(4)}\right), \\
c_{3}= & \varepsilon_{1}^{-1} \varepsilon_{2}^{-1} \gamma_{n_{1}} \gamma_{n_{2}}\left(\left(\varepsilon_{1} \gamma_{n_{2}}\left(1+\varkappa_{1} p_{1}^{(4)}+\varkappa_{1}\right)+\varepsilon_{2} \gamma_{n_{1}}\left(1+\varkappa_{2} p_{2}^{(4)}+\varkappa_{2}\right)\right.\right. \\
& \left.-\varkappa_{1} \varkappa_{2}\left(\gamma_{n_{1}} p_{1}^{(4)}+\gamma_{n_{2}} p_{2}^{(4)}+\gamma_{n_{1}}+\gamma_{n_{2}}\right)\right) p_{1}^{(4)} p_{2}^{(4)} \\
& \left.+\varepsilon_{2} \gamma_{n_{1}} p_{1}^{(4)}+\varepsilon_{1} \gamma_{n_{2}} p_{2}^{(4)}\right), \\
c_{4}= & \varepsilon_{1}^{-1} \varepsilon_{2}^{-1} \gamma_{n_{1}}^{2} \gamma_{n_{2}}^{2} p_{1}^{(4)} p_{2}^{(4)}\left(1+\varkappa_{1} p_{1}^{(4)}+\varkappa_{2} p_{2}^{(4)}-\varkappa_{1} \varkappa_{2}\left(p_{1}^{(4)}+p_{2}^{(4)}+1\right)\right) .
\end{aligned}
$$

Conditions (13) and (14) are always fulfilled. To analyze (15) and (16), we place $\varepsilon_{2}=\alpha \varepsilon_{2}, \gamma_{n_{2}}=\beta \gamma_{n_{2}}, j_{2}=\zeta j_{1}$ and $\varkappa_{2}=\eta \varkappa_{1}$, so that the auxiliary parameters $\alpha, \beta, \zeta$ and $\eta$ are within $O(1)$. In terms of this assumption, (15) can be boiled down, for $\varkappa_{1} \ll 1$, to

$$
\varepsilon_{1}^{-3} \gamma_{n_{1}}^{6}\left(m_{1} \varepsilon_{1}-m_{2} \varkappa_{1}^{2}\right)>0
$$

where $m_{1}$ and $m_{2}$ are positive coefficients depending only on $j_{1}$, such that $m_{1}=O\left(j_{1}^{4}\right)$ and $m_{2}=O\left(j_{1}^{5}\right)$. It can be seen that the above inequality and consequently (15) hold if

$$
\varkappa_{1}=o\left(\varepsilon_{1}^{1 / 2}\right) \text {. }
$$

As is known (15) guarantees a simple complex conjugate pair of eigenvalues corresponding to a linearization about steady state 4 to have negative real part.

Condition (16) can be shown to yield $\varkappa_{1}=o(1)$, however this constraint is weaker than (17). Thus (17) is the stability condition for steady state 4 .

Having regard to a fairly small value of $\varepsilon_{1}$, (17) may be thought to be broken under most physically meaningful conditions when coupling is not infinitesimally weak. Hence normally, condition (15) of the Routh-Hurwitz criterion is never fulfilled and "mixed" steady state 4, if any, is always unstable by growing oscillations. 
At not-too-weak coupling strengths, such that

$$
\varkappa_{1} \varkappa_{1}>1
$$

the system being studied is able to exhibit a hysteresis effect. (18) is obtained combining (11) and (12). Suppose, for definiteness, that we have a fixed $j_{2}=$ $j_{2}^{*}$, and $j_{1}$ increases from some value less than $j_{2}^{*} / \varkappa_{1}$ along the path $A B C D$ in the $j_{2}, j_{1}$ parameter space (Fig. 2a). Then, referring also to (10)-(12), we see that steady state 2 initially takes place at $A$ with laser 2 on and laser 1 off. This state remains unchanged with $j_{1}$ until $C$ in Fig. 2a is reached. For a larger $j_{1}$ steady state 2 gives up its stability and the system jumps to steady state 1 . Laser 2 becomes dim, while laser 1 takes over. If we now reduce $j_{1}$, the system is in steady state 1 and it remains there until $j_{1}$ reaches the lower critical value, where there is again only one stable steady state, at which there is a jump from steady state 1 to steady state 2 . In other words as $j_{1}$ increases along $A B C D$ there is a discontinuous switch from laser 2 to laser 1 at $C$ while as $j_{1}$ decreases from $D$ to $A$ there is a discontinuous switch from laser 2 to laser 1 at $B$. The hysteresis is made possible thanks to the concurrent stability of both "pure" equilibria on the interval from $B$ to $C$. In terms of electronics, such a situation would describe a flip-flop circuit having two stable conditions, each corresponding to one of two alternative input signals. If (18) is not met, then there are no stable steady states within $B C$. The hysteresis is an example of a cusp catastrophe which is illustrated schematically in Fig. $2 \mathrm{~b}$ where the letters $A, B, C$ and $D$ correspond to those in Fig. 2a. Note that Fig. 2a is the projection of the surface onto the $\left(j_{2}, j_{1}\right)$ plane with the wedge-shaped region corresponding to the overlap.

It is worth noting that the presence of the second-order loss in already mentioned model [8] leads to basically similar types of fixed points: two "pure" and two "mixed". However the fundamentally different coupling scheme induces a quite another bifurcational behavior of those steady states in regard to bias currents and coupling strengths.

\section{Two loss-coupled lasers: oscillatory dynamics}

When conditions (11) and (12) are not met, but instead,

$$
\left\{\begin{array}{l}
\varkappa_{1}<j_{2} / j_{1}, \\
\varkappa_{2}<j_{1} / j_{2},
\end{array}\right.
$$




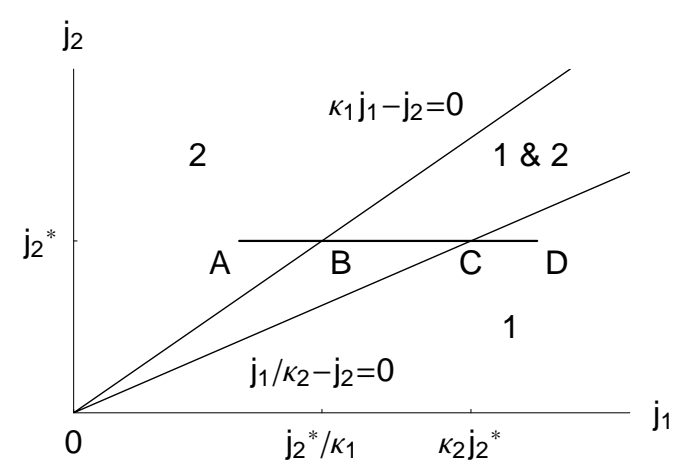

(a)

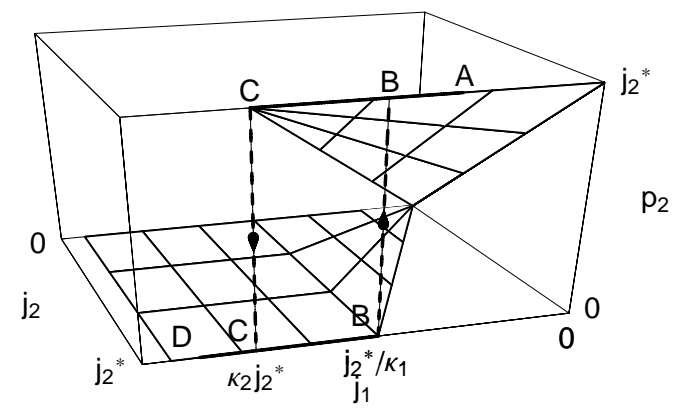

(b)

Fig. 2. (a) Parameter domain for the number of positive stable steady states for the model (8). Only the positive steady state 1 is stable below the line $j_{1} / \varkappa_{2}-j_{2}=0$. Similarly, the only stable steady state is 2 with its domain of existence above the line $\varkappa_{1} j_{1}-j_{2}=0$. Both states coexist in the region of bistability confined by the two straight lines, realization of either state being a matter of path-dependency. (b) A cusp catastrophe for the equilibrium states in the $\left(p_{2}, j_{2}, j_{1}\right)$ parameter space. As $j_{1}$ increases from $A$, the path is $A B C C D$ while as $j_{1}$ decreases from $D$, the path is $D C B B A$. The projection of the two surfaces onto the $\left(j_{2}, j_{1}\right)$ plane is given in (a). Two stable equilibria exist where the overlap is.

model (8) has three positive steady states, 2, 3 and 4, none of them being stable. "Mixed" steady state 4 is unstable by growing oscillations. In such a case the model would thus be expected to have a limit cycle in its four-dimensional phase space corresponding to sustained oscillations. At sufficiently weak coupling strengths of order $\varepsilon^{1 / 2}$, i. e. not too far away from the Hopf bifurcation, where condition (17) breaks down, this limit cycle is small and represents a low-amplitude quasi-harmonic periodic solution. As a practical matter, the range of such an extremely weak coupling is of less concern to us than is the range of far more feasible relatively strong coupling corresponding to welldeveloped substantially nonlinear oscillations. We are going to demonstrate that at not-too-weak coupling strengths and as conditions (19)take place, system (8) exhibits relaxation oscillatory behavior with the two coupled lasers being antiphase locked.

Since time constants for photons and carriers considerably differ, four-dimensional system (8) is singularly perturbed. Relatively fast variables are $p_{1}$ and $p_{2}$, and slow variables are $n_{1}$ and $n_{2}$. The standard practice of reducing such systems is adiabatical elimination of the fast variables, when the left-hand side in the fast equation is replaced by zero, thus turning this differential equation into an algebraic equation. It is assumed, that the fast variables quickly relax to their momentary equilibrium values obtained from the algebraic equations, in which the slow variables are treated as parameters. "Frozen" slow variables do not move substantially in this short adaptation time of the fast variables. The momentary equilibrium value of the fast variables can thereupon be expressed by value of the slow variable. The fast variables hastily adapt to the 
motion of the slow variables (order parameters). The former are entrained by the latter. Chemical physicists who were first to introduce this technique often refer to it as the quasi-steady-state approximation ( $Q S S A)$. The utility of the procedure is that it allows us to reduce the dimension of the system by retaining only order parameters in the model. One has to establish the validity of the adiabatical elimination in each specific case using the recommendations of the singular perturbation theory. In particular, Tikhonov theorem [10] requires quasi-steady state of the fast equations to be stable.

To replace the derivatives in the first two equations of (8),

$$
\left\{\begin{array}{l}
\dot{p}_{1}=\varepsilon_{1}^{-1} \gamma_{n_{1}}\left(n_{1}-\delta_{1} p_{1}-\varkappa_{2} p_{2}\right) p_{1} \\
\dot{p}_{2}=\varepsilon_{2}^{-1} \gamma_{n_{2}}\left(n_{2}-\delta_{2} p_{2}-\varkappa_{1} p_{1}\right) p_{2}
\end{array}\right.
$$

by zeros and reduce the respective equations to the algebraic system

$$
\left\{\begin{array}{l}
\left(n_{1}-\delta_{1} p_{1}-\varkappa_{2} p_{2}\right) p_{1}=0 \\
\left(n_{2}-\delta_{2} p_{2}-\varkappa_{1} p_{1}\right) p_{2}=0
\end{array}\right.
$$

in which $n_{1}$ and $n_{2}$ are treated as parameters, one has to ensure stability of quasi-steady states of the fast subsystem (20).

We anticipate the dynamics of singularly perturbed system (8) in the phase space $\left(p_{1}, p_{2}, n_{1}, n_{2}\right)$ to be consisted of two typical motions: quickly approaching the "slow" manifold (21) and slowly sliding along it until a leave point (where the solution disappears) is reached. After that the representing point possibly may jump to another local solution of (21).

Thus, we have to find all quasi-steady states of (20), distinguish the domains of their stability in the phase plane $\left(n_{2}, n_{1}\right)$ of the slow subsystem

$$
\left\{\begin{array}{l}
\dot{n}_{1}=\gamma_{n_{1}}\left(j_{1}-\left(n_{1}+1\right) p_{1}-n_{1}\right), \\
\dot{n}_{2}=\gamma_{n_{2}}\left(j_{2}-\left(n_{2}+1\right) p_{2}-n_{2}\right),
\end{array}\right.
$$

and then investigate the dynamics of the complete system (8) with piecewise continuous functions. 
Subsystem (20) has four quasi-steady states, two "pure" and two "mixed" (the slow variables are deemed to be "frozen"):

$$
\begin{aligned}
& p_{1}^{(\mathrm{qs} 1)}=n_{1} / \delta_{1}, \quad p_{2}^{(\mathrm{qs} 1)}=0 ; \\
& p_{1}^{(\mathrm{qs} 2)}=0, \quad p_{2}^{(\mathrm{qs} 2)}=n_{2} / \delta_{2} ; \\
& p_{1}^{(\mathrm{qs} 3)} \approx\left(\varkappa_{2} n_{2}-\delta_{2} n_{1}\right) /\left(\varkappa_{1} \varkappa_{2}\right), \\
& p_{2}^{(\mathrm{qs} 3)} \approx\left(\varkappa_{1} n_{1}-\delta_{1} n_{2}\right) /\left(\varkappa_{1} \varkappa_{2}\right) ; \\
& p_{1}^{(\mathrm{qs} 4)}=0, \quad p_{2}^{(\mathrm{qs} 4)}=0 .
\end{aligned}
$$

(Quasi-steady-state solution 3 is written in line with the assumption (9).)

"Pure" quasi-steady state 1 is a stable node everywhere below the line

$$
\varkappa_{1} n_{1}-\delta_{1} n_{2}=0
$$

in the parametric plane $n_{2}, n_{1}$ of the slow variables (Fig. 3a). It is realizable in two different phase portraits shown in Figs. 3b and 3c. By the same token "pure" quasi-steady state 2 is a stable node everywhere above the line

$$
\varkappa_{2} n_{2}-\delta_{2} n_{1}=0
$$

(Fig. 3a), and it is featured by the phase portraits in Figs. 3c and 3d.

Both "pure" quasi-steady states can coexist within the opening of the angle formed by lines (24) and (25) in Fig. 3a:

$$
\delta_{2} n_{1} / \varkappa_{2}<n_{2}<\varkappa_{1} n_{1} / \delta_{1} .
$$

The opening shrinks as coupling strengths get weaker. In this flip-flop domain the two "pure" quasi-steady states, both stable, are being separated by "mixed" quasi-steady state 3 of a saddle type (Fig. 3a).

As to "mixed" (trivial) quasi-steady state 4, it is always an unstable node.

Let us assume that "pure" quasi-steady state 1 is initially stable, the population inversions $n_{1}$ and $n_{2}$ are somewhere within the domain $\varkappa_{1} n_{1}-\delta_{1} n_{2}>0$, and also $n_{1}(0) \gg \delta_{1} j_{1}$. While $n_{1}$ remains much greater than $\delta_{1} j_{1}$, the dynamics of the slow variables (treated as bifurcation parameters in reference to the fast variables) is governed by a system of two independent equations

$$
\dot{n}_{1}=\gamma_{n_{1}}\left(j_{1}-n_{1}\left(n_{1}+1\right) / \delta_{1}-n_{1}\right)
$$




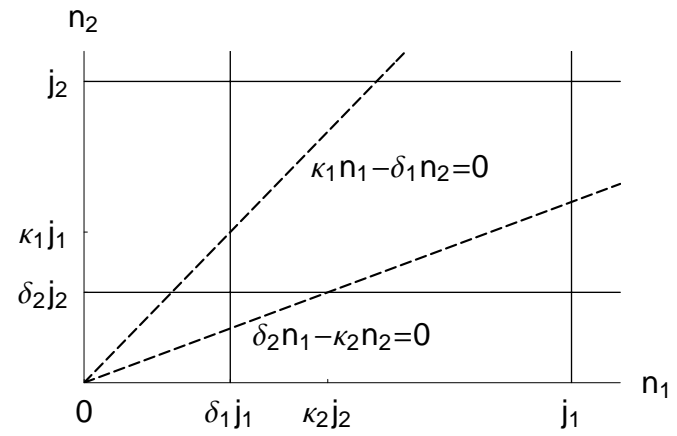

(a)

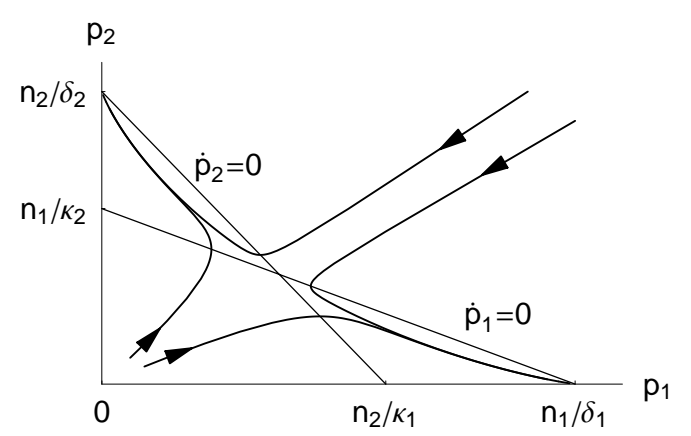

(c)

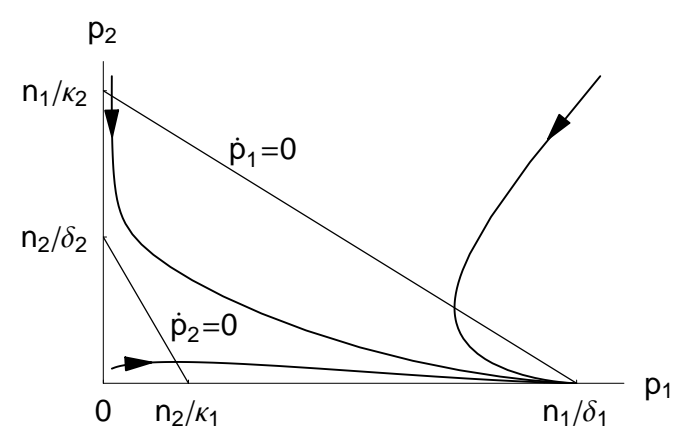

(b)

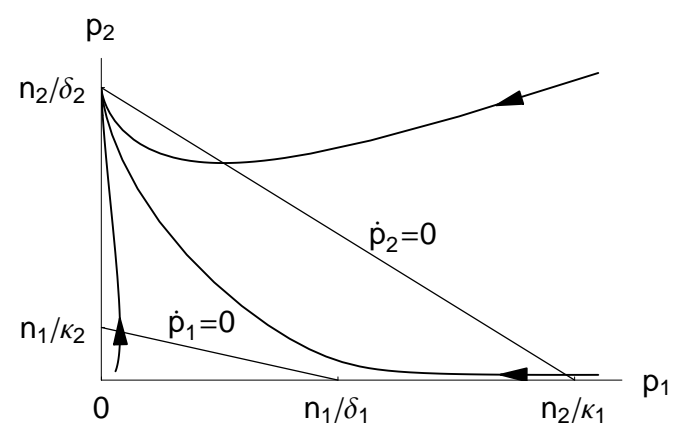

(d)

Fig. 3. (a) Phase plane of the slow variables (order parameters) $n_{1}$ and $n_{2}$ sectored (by dashed lines) into stability domains of the corresponding quasi-steady states of the fast subsystem (20). Both "pure" quasi-steady states, each corresponding to the situation when either of the two lasers is on, coexist within the opening of the angle formed by dashed lines (24) and (25). Lines $n_{1}=\delta_{1} j_{1}$ and $n_{2}=j_{2}$ are respective nullclines $\dot{n}_{1}=0$ and $\dot{n}_{2}=0$ of the piecewise system (27)-(28). Lines $n_{1}=j_{1}$ and $n_{2}=\delta_{2} j_{2}$ mean the same for the system (31)-(32). Intersections of the nullclines are equilibria of the associated piecewise slow subsystems, and they must be outside the abovementioned opening to allow for the relaxation oscillations. (b) Phase portrait of (20) for the case in which "pure" quasi-steady state 1 is the only stable solution. (c) Phase portrait of (20) for the flip-flop case in which both "pure" quasi-steady states are stable nodes and coexist being separated by a saddle point. Realization of either state depends on the initial conditions. (d) Phase portrait of (20) for the case in which "pure" quasi-steady state 2 is the only stable solution.

$$
\dot{n}_{2}=\gamma_{n_{2}}\left(j_{2}-n_{2}\right) \text {. }
$$

This system has (stable) steady state

$$
n_{1}^{(1)}=\delta_{1} j_{1}+O\left(\delta_{1}^{2}\right), \quad n_{2}^{(1)}=j_{2},
$$

and the representing point will tend to reach it. System (27)-(28) also allows for distinguishing fast and slow variables. Due to small value of $\delta_{1}$, equation (27) is roughly $\delta_{1}^{-1}$ faster than equation (28). Therefore $n_{1}$ relatively quickly 


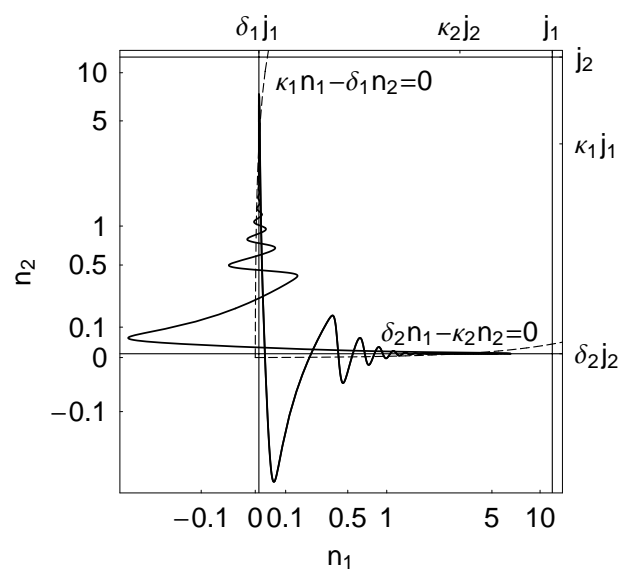

Fig. 4. Logarithmically stretched-out neighborhood of point $\left(\delta_{1} j_{1}, \delta_{2} j_{2}\right)$ in the phase plane of the order parameters (cf. Fig. 3a) and the projection of the limit cycle. In this numerical example parameters of the two lasers are identical with the exception of the pumps chosen to be $j_{1}=11.9$ and $j_{2}=12.5$. Coupling strengths are $\varkappa_{1}=0.30$ and $\varkappa_{2}=0.25$. Note transverse damped vibrations of the representing point whenever it moves along either of two nullclines $\dot{n}_{1}=0$ and $\dot{n}_{2}=0$.

relaxes to $n_{1}^{(1)}, n_{2}$ being practically "frozen". In other words, the representing point first arrives at the "slow" nullcline $\dot{n}_{1}=0$, given by $n_{1} \approx \delta_{1} j_{1}$. If $\delta_{1}$ were large enough to damp the intrinsic oscillations of laser 1, the representing point would further slowly slide along that nullcline, calmly tending to $j_{2}$. Still small value of $\delta_{1}$ complicates the picture, and in the immediate vicinity of $n_{1}^{(1)}$ the system gets trapped into a stable focus with respect to variables $p_{1}$ and $n_{1}$. Here QSSA ceases to be true, and one can no longer substitute $p_{1}$ by its quasi-steady-state value $p_{1}^{\text {(qss1) }}$. Instead of (27) we have to write down a pair of equations

$$
\left\{\begin{array}{l}
\dot{p}_{1}=\varepsilon_{1}^{-1} \gamma_{n_{1}}\left(n_{1}-\delta_{1} p_{1}\right) p_{1}, \\
\dot{n}_{1}=\gamma_{n_{1}}\left(j_{1}-\left(n_{1}+1\right) p_{1}-n_{1}\right) .
\end{array}\right.
$$

System (30) is identical to the rate equations (3) of an uncoupled laser and in essence describes underdamped intrinsic oscillations. In the plane of the slow variables these oscillations manifest themselves in damped transverse fluctuations superimposed on the independent vertical motion along the nullcline $\dot{n}_{1}=0$ toward $n_{2}^{(1)}=j_{2}$ (Fig. 4 ). Thus, $n_{2}$ is the actual order parameter in this area of the complete four-dimensional phase space. If steady state 1 (given by (29)) lies beyond the area $\varkappa_{1} n_{1}-\delta_{1} n_{2}>0$ (Fig. 3a), or the same, if the first inequality of (19) holds true, then the representing point would inevitably touch the upper boundary of the domain (26) at a point $\left(\delta_{1} j_{1}, \varkappa_{1} j_{1}\right)$ before approaching the vicinity of steady state 1 . On the boundary given by equation (24), "pure" quasi-steady state 1 for the entrained variables merges with "mixed" saddle quasi-steady state 3 and loses its stability. Laser 1 instantly 
switches off, and the alternative "pure" quasi-steady state 2 becomes stable, with laser 2 being on.

In terms of four-dimensional phase space of the complete system (8), the representing point is now in the other stable hyperplane of the "slow" manifold (21). Slow subsystem sliding along this alternative branch obeys the equations

$$
\begin{aligned}
& \dot{n}_{1}=\gamma_{n_{1}}\left(j_{1}-n_{1}\right), \\
& \dot{n}_{2}=\gamma_{n_{2}}\left(j_{2}-n_{2}\left(n_{2}+1\right) / \delta_{2}-n_{2}\right),
\end{aligned}
$$

with the initial conditions $n_{1}(0)=n_{1}^{(1)}=\delta_{1} j_{1}$ and $n_{2}(0)=\varkappa_{1} j_{1}$. System (31)-(32) has steady state

$$
n_{1}^{(2)}=j_{1}, \quad n_{2}^{(2)}=\delta_{2} j_{2}+O\left(\delta_{2}^{2}\right),
$$

that is stable. Variable $n_{2}$ quickly approaches the nullcline $\dot{n}_{2}=0$ given by $n_{2} \approx \delta_{2} j_{2}$, and then starts to oscillate about it according to the equations

$$
\left\{\begin{array}{l}
\dot{p}_{2}=\varepsilon_{2}^{-1} \gamma_{n_{2}}\left(n_{2}-\delta_{2} p_{2}\right) p_{2}, \\
\dot{n}_{2}=\gamma_{n_{2}}\left(j_{2}-\left(n_{2}+1\right) p_{2}-n_{2}\right) .
\end{array}\right.
$$

Equations (34) describe underdamped intrinsic oscillations of laser 2. At the same time $n_{1}$ (which is now the order parameter) relatively slowly tends to $j_{1}$ along the nullcline $\dot{n}_{2}=0$. Again, if steady state (33) is located below line (25), or equally, if the second inequality of (19) holds true, then the representing point would certainly touch boundary $(25)$ at a point $\left(\delta_{2} j_{2}, \varkappa_{2} j_{2}\right)$, whereupon "pure" quasi-steady state 2 for the fast variables would merge with saddle quasi-steady state 3 without fail and lose its stability in favor of "pure" quasisteady state 1 . The system returns to the first branch, and the oscillatory cycle gets closed.

\section{Discussion}

Thus, under conditions (19) system (8) features sustained relaxation oscillations (Fig. 5). The coupled lasers turn out to be antiphase locked. In its principle of operation, the considered system resembles astable multivibrator, known to be an electronic circuit that oscillates between its two states, neither of which is stable, generating a continuous flow of square edge pulses.

It should be mentioned that quite similar antiphase synchronization patterns have been previously found (both numerically and experimentally) by T. Baer 


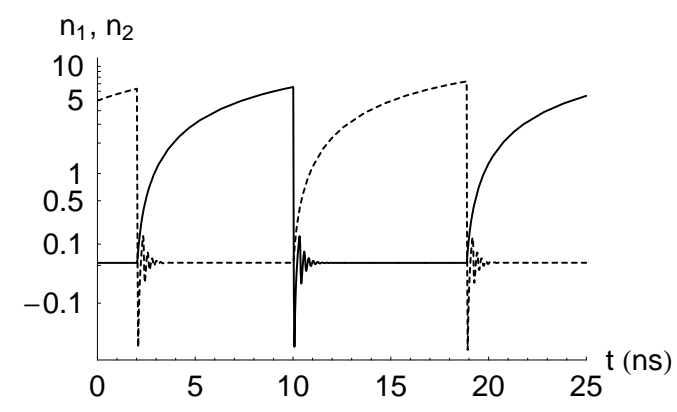

(a)

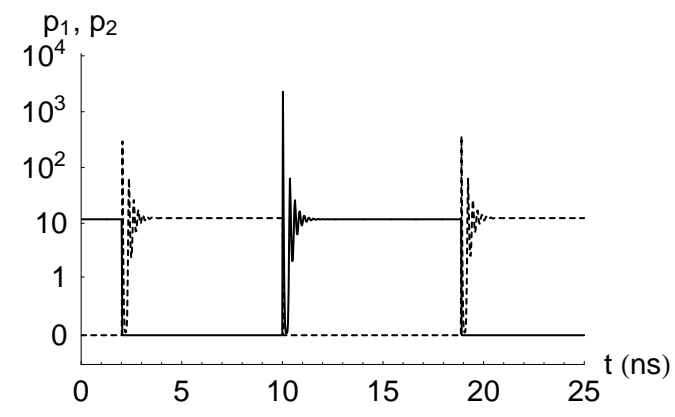

(b)

Fig. 5. Time profiles of oscillating population inversions (a) and photon numbers (b) in two loss-coupled lasers. Solid: laser 1, dashed: laser 2. Numerical values of the parameters are those mentioned in the caption to Fig. 4.

[11] and K. Wiesenfeld, C. Bracikowski, G. James and R. Roy [12] in a different system featured by two coupled longitudinal modes oscillating in Nd:YAG laser with an intracavity-doubling crystal.

The population inversions, $n_{1}$ and $n_{2}$, demonstrate saw-tooth periodical pulses. Oscillation range for the population inversions remains finite, and, what is important, does not depend on $\delta_{1}$ and $\delta_{2}$. The respective amplitudes for $n_{1}$ and $n_{2}$ are of orders of $\varkappa_{2} j_{2}$ and $\varkappa_{1} j_{1}$.

Photon numbers, $p_{1}$ and $p_{2}$, inside the cavities change periodically between quiescence and short giant spikes. The magnitude of power output spikes, in contrast to carrier's pulses, tends to infinity as $\delta_{1,2} \rightarrow 0$, in view of equations (23).

Times of motion over the either branch of the "slow" manifold (21), $\tau_{1}$ and $\tau_{2}$, add up to give a period of oscillations, $T$. Those times are predominantly determined by dynamics of the order parameters $n_{1}$ and $n_{2}$, and, to a zeroth approximation in $\varepsilon_{1}$ and $\varepsilon_{2}$, can be found as solutions of the equations of motion (31) and (28) with respective boundary conditions $n_{1}(0)=0, n_{1}\left(\tau_{1}\right)=\varkappa_{2} j_{2}$, and $n_{2}(0)=0, n_{2}\left(\tau_{2}\right)=\varkappa_{1} j_{1}$. Therefore we obtain a quite simple estimate for the period:

$$
\begin{aligned}
T & =\tau_{1}+\tau_{2}=\gamma_{n_{1}}^{-1} \int_{0}^{\varkappa_{2} j_{2}} \frac{d \xi}{j_{1}-\xi}+\gamma_{n_{2}}^{-1} \int_{0}^{\varkappa_{1} j_{1}} \frac{d \xi}{j_{2}-\xi} \\
& =\gamma_{n_{1}}^{-1} \ln \frac{1}{1-\varkappa_{2} j_{2} / j_{1}}+\gamma_{n_{2}}^{-1} \ln \frac{1}{1-\varkappa_{1} j_{1} / j_{2}}
\end{aligned}
$$

The typical temporal scale of the oscillations is determined by carrier population inversion time constants and turns to be measured in nanoseconds (which is much longer than underdamped oscillations of a single laser). It is interesting, that according to (35) the period depends on the ratio of the pump 


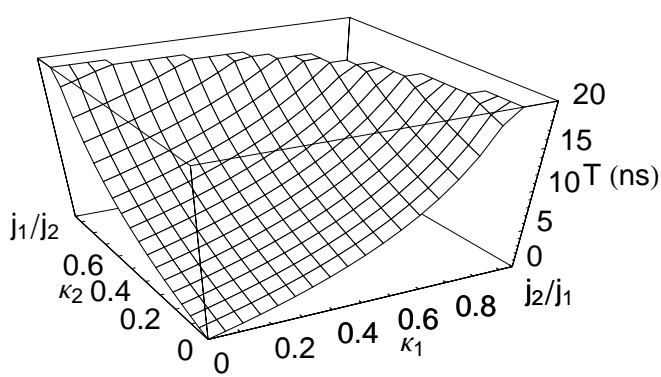

(a)

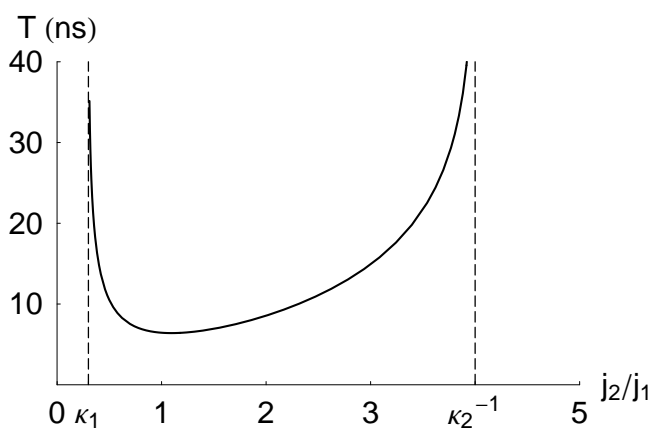

(b)

Fig. 6. The period of synchronous oscillations versus coupling strengths (a) and pumps ratio (b). Numerical values of the parameters are those mentioned in the caption to Fig. 4.

currents, $j_{2} / j_{1}$, rather than on each of the two currents individually, and completely does not depend on concrete values of $\delta_{1}$ and $\delta_{2}$. At weak coupling the period linearly shortens, but tends to infinity whenever either $\varkappa_{1}$ or $\varkappa_{2}^{-1}$ approaches $j_{2} / j_{1}$ (Fig. 6a). Given $\varkappa_{1}$ and $\varkappa_{2}$, such that $\varkappa_{1} \varkappa_{2}<1$, the ratio $j_{2} / j_{1}$ has to be confined between $\varkappa_{1}$ and $\varkappa_{2}^{-1}$ (Fig. 6b). Within that area the period relatively weakly depends on the ratio of the two pumps.

In all likelihood, the limit cycle would persist in our model of coupled lasers even if the second-order cavity loss is not merely small but absent whatsoever. However in such a case the quasi-steady state approximation technique is inapplicable since at $\delta_{1,2}=0$ fast subsystem (20) has no finite "pure" equilibria. Generally speaking, as $\delta_{1,2}$ becomes of order $\varepsilon_{1,2}$ equations (27) and (32) can no longer be considered slow and the approximate estimate (35) loses its accuracy. QSSA yields its best accuracy for $\varepsilon_{1,2} \ll \delta_{1,2} \ll 0$.

Earlier, B.A. Nguyen and P. Mandel [3] have shown (in the framework of their model) that equally pumped lasers become unstable for much smaller values of the loss cross-coupling than unequally pumped lasers. Their result has to do with the onset of oscillations. Our model deals with well developed highamplitude nonlinear oscillations and therefore allows to predicts somewhat similar related to quenching: synchronous oscillations in equally pumped lasers are being quenched for smaller values of the greater of two coupling strengths than in unequally pumped lasers. Indeed, it follows from conditions (19) that if pumps are equal, $j_{1}=j_{2}$, then both coupling strengths, $\varkappa_{1}$ and $\varkappa_{2}$, must be less than unity for the synchronous oscillations to go on. In case of the different pumps, however, one of the coupling strengths may be greater than unity.

The most intriguing feature of the considered model is that each of the two lasers by itself does not lase, however in interaction, when coupled in a nonlinear way, the resulting system is shown to have sustained oscillations. As 
far back as in early 1970s, S. Smale [13] constructed an abstract mathematical example of a cell modeled by the chemical kinetics of four metabolites, $x_{1}, \cdots, x_{4}$, such that the reaction equations $\dot{\mathbf{x}}=\mathbf{R}(\mathbf{x})$ for the set of metabolites, $\mathbf{x}=\left(x_{1}, \cdots, x_{4}\right)$, had a globally stable equilibrium. The cell is "dead" in that the concentrations of its metabolites always tend to the same fixed levels. When two such cells are coupled by linear diffusion terms of the form $\mathbf{M}\left(\mathbf{x}_{2}-\mathbf{x}_{1}\right)$, where $\mathbf{M}$ is a diagonal matrix with the elements $\mu_{k} \delta_{k l}$, however, the resulting equations are shown to have a globally stable limit cycle. The concentrations of the metabolites begin to oscillate, and the system becomes "alive". In Smale's words:

There is a paradoxical aspect to the example. One has two dead (mathematically dead) cells interacting by a diffusion process which has a tendency in itself to equalize the concentrations. Yet in interaction, a state continues to pulse indefinitely.

Smale also remarks that 'it is more difficult to reduce the number of chemicals to two or even three.'

Equations (8) may be interpreted in biological terms if we assume $p_{1}, p_{2}$ and $n_{1}, n_{2}$ to be respectively species (predators) and nutrients (preys) consumed by the species. The corresponding nutrients are fed into the system with some constant rates $j_{1}$ and $j_{2}$. In such a case the model describes interference interspecific competition between two predators belonging to different species competition that does not act through the utilization of a nutritious resource, but instead involves direct interaction between the competitors (e.g. through aggressive behavior). Coupling strengths $\varkappa_{1}$ and $\varkappa_{2}$ just reflect the intensity of interspecific competition. As distinct from the Smale's example, coupling is nonlinear and this makes sustained synchronous oscillations possible for fewer number of variables. Constants $\delta_{1}$ and $\delta_{2}$ (sometimes referred to as Verhulst parameters in ecology) are responsible for intraspecific competition resulting in a reduction of population growth rate as population density increases. Unfortunately the ecological analogy is limited because of at least two important reasons.

First, species usually influence other species less strongly than they do their conspecifics, in other words, interspecific competition is typically dominated by intraspecific competition:

$$
\varkappa_{1,2}<\delta_{1,2}
$$

In our case of loss-coupled lasers, second-order cavity losses are almost negligible in comparison with the coupling (recall (9)). As early as in 1930s G.F. Gause and A.A. Witt [14] considered two competing species described by a pair of equations like (20) with constant $n_{1}$ and $n_{2}$ (which implies the abun- 
dance of food resources) and showed that when conditions (36) are met, the two species would coexist. In our model the two photon populations neither coexist concurrently, nor exclude each other forever. Nevertheless we can say that the two populations coexist in different temporal niches, in the manner of time sharing.

Second, time scales are usually inverted in ecosystems as opposed to laser. That is to say, food is consumed by species rapidly, i.e.

$$
\gamma_{n_{1,2}} \gg \gamma_{p_{1,2}}
$$

is the common case in ecology. In our model slowness of carrier population relative to photon population is essential for the oscillations to occur because it provides the necessary inertia to the system.

It is notable in this connection that recently M.-Y. Kim, R. Roy, J.L. Aron, T.W. Carr and I.B. Schwartz [1c] have found a case of complete analogy between coupled lasers and coupled living populations. It is shown that the rate equations for two lasers coupled optoelectronically through their pump currents are identical to the rate equations for two migration-coupled infective human populations. From the ecological perspective, the model being discussed and ours describe different types of competition. Model [1c] corresponds to trophic interspecific competition, because competitors are allowed to affect food sources of each other. In that model coupling is brought about through a slow variable (carriers/susceptible individuals), while in model (8) the lasers are coupled through a fast variable (photons). For this reason the two models exhibit different behavior. In particular, the presence of time delay in coupling is prerequisite to quasi-harmonic self-pulsing regime in model [1c]. Synchronous anti-phase oscillations in our model do not require such a delay, although introducing it to the equations has much potential for further studying the system.

The author expresses his gratitude to R. Roy and I.B. Schwartz for fruitful discussion.

\section{References}

[1a] G. Giacomelli, M. Calzavara and F.T. Arecchi, Opt. Comm. 74 (Dec.) (1989) $97-101$.

[1b] S. Tang, R. Vicente, M.C. Chiang, C.R. Mirasso and J.-M. Liu, IEEE J. of Select. Topics in Quant. Electron. 10 (5) (2004) 936-943.

[1c] M.-Y. Kim, R. Roy, J.L. Aron, T.W. Carr and I.B. Schwartz, Phys. Rev. Lett. 94 (2005) 088101. 
[2a] J. Javaloyes, P. Mandel and D. Pieroux, Phys. Rev. E 67 (2003) 036201.

[2b] F. Rogister and J. García-Ojalvo, Opt. Lett. 28 (14) (2003) 1176-1178.

[2c] S. Yanchuk, K.R. Schneider and L. Recke, Phys. Rev. E 69 (2004) 056221.

[3] B.A. Nguyen and P. Mandel, J. Opt. B 1 (1999) 320-324.

[4] C.L. Tang, H. Statz and G. deMars, J. Appl. Phys. 34 (8) (1963) 2289-2295.

[5] G.P. Agrawal and N.K. Dutta, Semiconductor Lasers (Van Nostrand Reinhold, New York, 1993), ch. 6.

[6] An unhappy term "relaxation oscillations" is quite often used in reference to those quasi-harmonic oscillations. For the avoidance of confusion, it seems reasonable to reserve this term to highly nonlinear discontinuous (jerky) oscillations.

[7] C.J. Kennedy and J.D. Barry, IEEE J. Quant. Electron. QE-10 (8) (1974) 596-599.

[8] R. Vicente, S. Tang, J. Mulet, C.R. Mirasso and J.-M. Liu, Phys. Rev. E 70 (2004) 046216.

[9] R.E. O'Malley, Introduction to Singular Perturbations (Academic Press, New York, 1974).

[10] A.N. Tikhonov, Matematicheskii sbornik, 32(73) (3) (1952) 575-586 (in Russian).

[11] T. Baer, J. Opt. Soc. Amer. 3 (9) (1986) 1175-1180.

[12] K. Wiesenfeld, C. Bracikowski, G. James and R. Roy, Phys. Rev. Lett. 65 (14) (1990) 1749-1752.

[13] S. Smale, in: J.D. Cowan, ed., Some Mathematical Questions in Biology, V: AMS Lecture Notes on Mathematics in the Life Sciences, vol. 6, (Amer. Math. Soc., Providence, RI, 1974), 15-26.

[14] G.F. Gause and A.A. Witt, Amer. Naturalist 69 (725) (1935) 596-609. 